\title{
40 años de estructuras de suelo reforzadas con geomalla de polietileno de alta densidad (HDPE): Los muros más altos de Latinoamérica a los Emiratos Árabes Unidos
}

DOI: $10.46932 / \mathrm{sfjdv2n2-126}$

Received in: March 1st, 2021

Accepted in: May 30th, 2021

\author{
C. Doulala-Rigby \\ Tensar International Limited \\ E-mail: CRigby@tensar.co.uk \\ A. Pérez \\ Ingnova \\ E-mail: ingnovadora@gmail.com
}

\section{RESUMEN}

El primer uso del refuerzo de geomalla polimérico de polietileno de alta densidad (HDPE) en ingeniería civil fue reforzar y construir un muro de suelo reforzado provisional de 2,5 $\mathrm{m}$ de alto en la fábrica de carbón Newmarket / Silkstone en West Yorkshire, Reino Unido en 1980, justo un año después de que la primera geomalla polimérica fuera inventada por el Dr. Mercer en 1979 en Blackburn, Reino Unido. Desde entonces, los sistemas de muros de retención de suelo reforzado (RSRW, por sus siglas en inglés) se han utilizado ampliamente en todo el mundo para formar diversas geometrías, alcanzar alturas cada vez mayores y cumplir diversas funciones, desde campos de golf al aire libre hasta pistas de aeropuertos. Este documento ofrece una visión histórica de cómo han evolucionado los sistemas RSRW en los últimos 40 años, sus componentes y mecanismos básicos, y presentará los estudios de caso de 2 muros de suelo reforzado de gran altura, construidos con geomallas poliméricas de polietileno de alta densidad. Un muro en Guatemala de 20m de altura y otro de 60m (escalonado) de altura en los Emiratos Árabes Unidos.

Palabras clave: suelo reforzado, geomalla polimérica, HDPE, muro de contención.

\begin{abstract}
The first use of High Density Polyethylene (HDPE) polymeric geogrid reinforcement in civil engineering was to reinforce and construct a $2.5 \mathrm{~m}$ high temporary reinforced soil wall at Newmarket/Silkstone colliery in West Yorkshire, UK in 1980, just a year after the first polymeric geogrid was invented by Dr Mercer in 1979 in Blackburn, UK. Reinforced Soil Retaining Wall (RSRW) Systems have since been widely used around the world forming various geometries, reaching ever growing heights and serving various functions from supporting open air golf courses to airport runways. This paper gives a historic insight on how RSRW Systems have evolved in the past 40 years, their basic components and mechanism and will present the case studies of Tensar's 2 tallest reinforced soil walls constructed with HDPE polymeric geogrids and locally sourced site won reinforced fill reaching heights up to $20 \mathrm{~m}$ in Guatemala and $60 \mathrm{~m}$ (cumulative) height in the United Arab Emirates respectively.
\end{abstract}

Keywords: reinforced soil, polymeric geogrid, HDPE, retaining wall 


\section{INTRODUCCIÓN}

La primera geomalla polimérica HDPE con uniones integrales fue inventada y patentada por el Dr. Mercer en 1979 en Blackburn, Reino Unido. Un año más tarde, y si bien todavía no había códigos de diseño oficiales disponibles, el primer uso de este refuerzo de geomallas HDPE fue la construcción un muro de suelo reforzado temporal de 2,5 m de altura en Newmarket / Silkstone Colliery en West Yorkshire, Reino Unido en 1980, utilizando residuos de la mina como material de relleno (Jones, 2014).

En los últimos 40 años se ha aprendido mucho sobre las propiedades de las geomallas poliméricas en usos de ingeniería. Muchas geomallas poliméricas han salido al mercado por varios fabricantes, cada uno funcionando de una manera única.

Del mismo modo, una gran cantidad de estándares y códigos de diseño se formularon para capturar el mecanismo de diseño y cálculo de la interacción entre el suelo y el refuerzo de geomalla. La publicación del Estándar Británico BS 8006 en 1995, después de haber estado en preparación desde 1984, cuando se formó el primer comité de estándares británico sobre suelo reforzado.

La Administración Federal de Carreteras de Estados Unidos de América (FHWA por sus siglas en inglés) publicó “Guidelines for Design, Specification, and Contracting of Geosynthetic Mechanically Stabilized Earth Slopes on Firm Foundations” en 1993 y luego otros países como Alemania, Holanda, Australia, Hong Kong, Sudáfrica y otros siguieron con sus propias publicaciones de estándares de diseño. El primer Simposio sobre geomallas poliméricas que se organizó tuvo lugar en 1982 bajo los auspicios de la Institución de Ingenieros Civiles en el Reino Unido.

Desde entonces, la tecnología de suelo reforzado con geomalla polimérica HDPE se ha utilizado en la construcción de miles de estructuras, incluidos terraplenes para ferrocarriles, carreteras principales y autopistas, así como muros marinos, aeropuertos, estribos de puentes, etc.

Este documento se centrará en las características principales que deben abordarse al utilizar la tecnología de suelo reforzado con geomallas de HDPE, sus componentes principales y las consideraciones de diseño y construcción que rigen su implementación exitosa.

El documento también presenta dos estudios de caso que muestran los 2 muros de contención de gran altura donde se utilizó suelo del lugar para el suelo reforzado de relleno y geomallas poliméricas de HDPE. 


\section{CARACTERÍSTICAS PRINCIPALES DE LOS SISTEMAS RSRW}

\subsection{COMPONENTES DE DISEÑO}

Los sistemas de muros de retención de suelo reforzado (RSRW, por sus siglas en inglés) comprenden cuatro componentes principales; la fachada, la geomalla uniaxial de HDPE, la conexión entre la geomalla y la fachada y el material de relleno reforzado.

La fachada pueden ser unidades modulares de concreto, paneles de concreto, paneles de altura completa, paneles de malla de acero, cajas de gaviones, o simplemente envolturas de geomallas biaxiales de polipropileno.

Las geomallas de polietileno de alta densidad (HDPE) están orientadas uniaxialmente y se les deben realizar pruebas de acuerdo con estándares reconocidos internacionalmente, como ISO y ASTM.

La conexión polimérica entre la geomalla y el revestimiento puede ser un conector mecánico de polímero para paredes de concreto modulares, que también están hechas de HDPE para proporcionar un alto nivel de transferencia de carga en la conexión, un conector mecánico en algunas patentes de bloques segmentados o una envoltura polimérica que conecta geomalla a geomalla, geomalla a panel de malla de acero o geomalla a caja de gavión.

Por último, el material de relleno reforzado es el componente más importante en cualquier estructura de suelo reforzado. Dependiendo del tipo, tamaño, ubicación y función final de cada estructura de suelo reforzado, el suelo reforzado que se puede usar puede variar desde un relleno granular bien graduado hasta un material cohesivo del sitio, tiza (Doulala-Rigby,2011), ceniza pulverizada (DoulalaRigby,2015), agregados ligeros (Doulala-Rigby,2017), poliestireno expandido e incluso material de relleno de residuos (Doulala-Rigby,2011). Los sistemas de muros de contención reforzados con geomalla se han utilizado con diferentes tipos de relleno de refuerzo durante 40 años. La principal limitación para elegir el relleno reforzado apropiado es su especificación precisa y los parámetros de resistencia al corte que se derivan de las pruebas apropiadas y garantizar que el material de relleno reforzado disponible para la construcción en el sitio cumpla con los requisitos de diseño y posteriormente, se coloque y compacte cumpliendo lo especificado en el diseño.

\subsection{MÉTODOS DE DISEÑO}

Al igual que para cualquier otra estructura de retención, el diseño debe incluir dos controles: estabilidad externa y estabilidad interna. Los controles de estabilidad externos comprenden los chequeos de deslizamiento, capacidad portante y volteo, y siguen el mismo principio que en un diseño de muro de contención por gravedad. La estabilidad externa proporciona la geometría del "bloque" reforzado con la geomalla, es decir, la longitud del refuerzo de la geomalla. La masa del bloque de suelo reforzado 
proporciona la fuerza restrictiva para deslizarse con la fuerza de empuje que proviene de la cuña activa en el suelo retenido. También se debe chequear la capacidad portante. Finalmente, se calcularán los asentamientos para evaluar si no exceden los límites especificados.

La estabilidad interna comprende dos controles fundamentales: falla por ruptura y falla por extracción de geomalla o verificación de adherencia. La estabilidad interna proporciona la resistencia y el espaciamiento de la geomalla que se requieren para una estructura estable. Los factores de interacción se utilizan para el deslizamiento y la extracción y se derivan de pruebas exhaustivas realizadas entre las geomallas y varios tipos de rellenos. Cuando se propone un nuevo tipo de relleno, o un material de relleno altamente arcilloso para ser utilizado como relleno reforzado, se debe llevar a cabo una prueba de extracción específica para el proyecto. Dependiendo de la norma internacional que se utiliza para llevar a cabo el diseño de los muros de suelo reforzado, la estabilidad interna se puede llevar a cabo basándose en dos principios diferentes: el método de diseño de cuña de 2 partes y el método de diseño de cuña de 1 parte o también llamado "Tie Back". El método de diseño de cuña en dos partes para materiales de refuerzo geosintéticos se utiliza principalmente en Alemania (EBGEO) y Australia (AS4678). El método de diseño de "Tie Back" o de 1 parte se utiliza principalmente en el Reino Unido, Estados Unidos y Hong Kong. Ambos métodos verifican la estabilidad interna del bloque de suelo reforzado, es decir, la verificación de la falla por extracción de las geomallas, así como la falla contra la ruptura de la geomalla, pero considerando diferentes modelos de falla. Ambos métodos también chequean el deslizamiento a lo largo de la base de todo el bloque de suelo reforzado y la capacidad de carga utilizando la distribución de presiones de Meyerhof.

La estabilidad global (general) de los muros también se lleva a cabo típicamente con el método simplificado de Bishop en un análisis de equilibrio límite. Una vez que se satisface la estabilidad externa e interna y se calcula la longitud, el tipo y el espaciado de las geomallas, el diseño final se transfiere a los planos de construcción como secciones, plantas, elevaciones y cualquier otro detalle específico del proyecto.

\subsection{CONSTRUCCIÓN}

La construcción de un RSRW es un procedimiento relativamente sencillo con todos los componentes colocados de acuerdo con la secuencia de construcción apropiada, bien establecida y probada. En resumen, las geomallas uniaxiales, caracterizadas por aberturas y nervaduras largas y delgadas formadas por material HDPE virgen estirado en una dirección durante la fabricación para producir una geomalla con una resistencia significativamente mayor en la dirección de la máquina se colocan perpendiculares al muro y se aseguran a cualquier revestimiento que se elija con la técnica y 
conexión adecuada. El relleno reforzado se coloca en capas de $150 \mathrm{~mm}$ a $300 \mathrm{~mm}$ (máx.) bien compactado, generalmente hasta el $95 \%$ de su densidad seca. La geomalla se coloca entre las capas de relleno compactadas a una separación vertical que varía típicamente de $150 \mathrm{~mm}$ a $600 \mathrm{~mm}$.

\section{ESTUDIOS DE CASO}

Desde la construcción del primer muro de contención de polietileno de HDPE en Silkstone Quarry en 1980 en el Reino Unido, la tecnología de muros de retención de suelo reforzado con geomalla polimérica ha alcanzado un amplio alcance en todo el mundo. Los dos estudios de caso que se presentan a continuación representan el muro de mayor altura de su clase que se ha construido en Guatemala y el muro de retención de suelo reforzado más alto del mundo jamás construido con geomalla polimérica de Tensar y se encuentra en Fujairah UAE.

\subsection{MUROS DE CONTENCIÓN DE SUELO REFORZADO DE 20 M DE ALTURA EN GUATEMALA.}

Los RSRW se han venido utilizando en Guatemala desde los años noventa y han tenido un gran auge en los últimos años. Al inicio fueron utilizados en proyectos residenciales de pequeñas dimensiones, hoy en día podemos apreciar rampas de aproximación en puentes, estribos verdaderos y muros de tamaño considerable y geometrías complejas.

El crecimiento urbano ha llevado a los desarrolladores a alejarse de la planicie del valle de la ciudad de Guatemala y han llevado sus proyectos a sitios con pendientes pronunciadas o zonas donde los muros de retención son una necesidad.

Para los muros de hasta 3.00 metros de altura lo más común sigue siendo el uso de muros de mampostería reforzada o concreto reforzado, pero mediante la altura aumenta, estas tipologías estructurales dejan de ser viables por lo que los muros reforzados con geomallas se han convertido en una necesidad.

En el año 2015, durante uno de estos desarrollos deportivos-habitacionales surgió un relleno que necesitaba como confinamiento un muro de grandes dimensiones. Luego de buscar la geometría que mejor se acoplara a las necesidades del desarrollador se llegó a un muro escalonado de 20 metros de altura total, dividido en dos secciones casi verticales a $70^{\circ}$ de inclinación con una berma intermedia de 5.00 metros de ancho en promedio. Este muro con sus 20 metros de alto y un área de fachada de 2850 metros cuadrados, es el más alto y de mayor tamaño en Guatemala y uno de los más grandes construidos en Latinoamérica. 

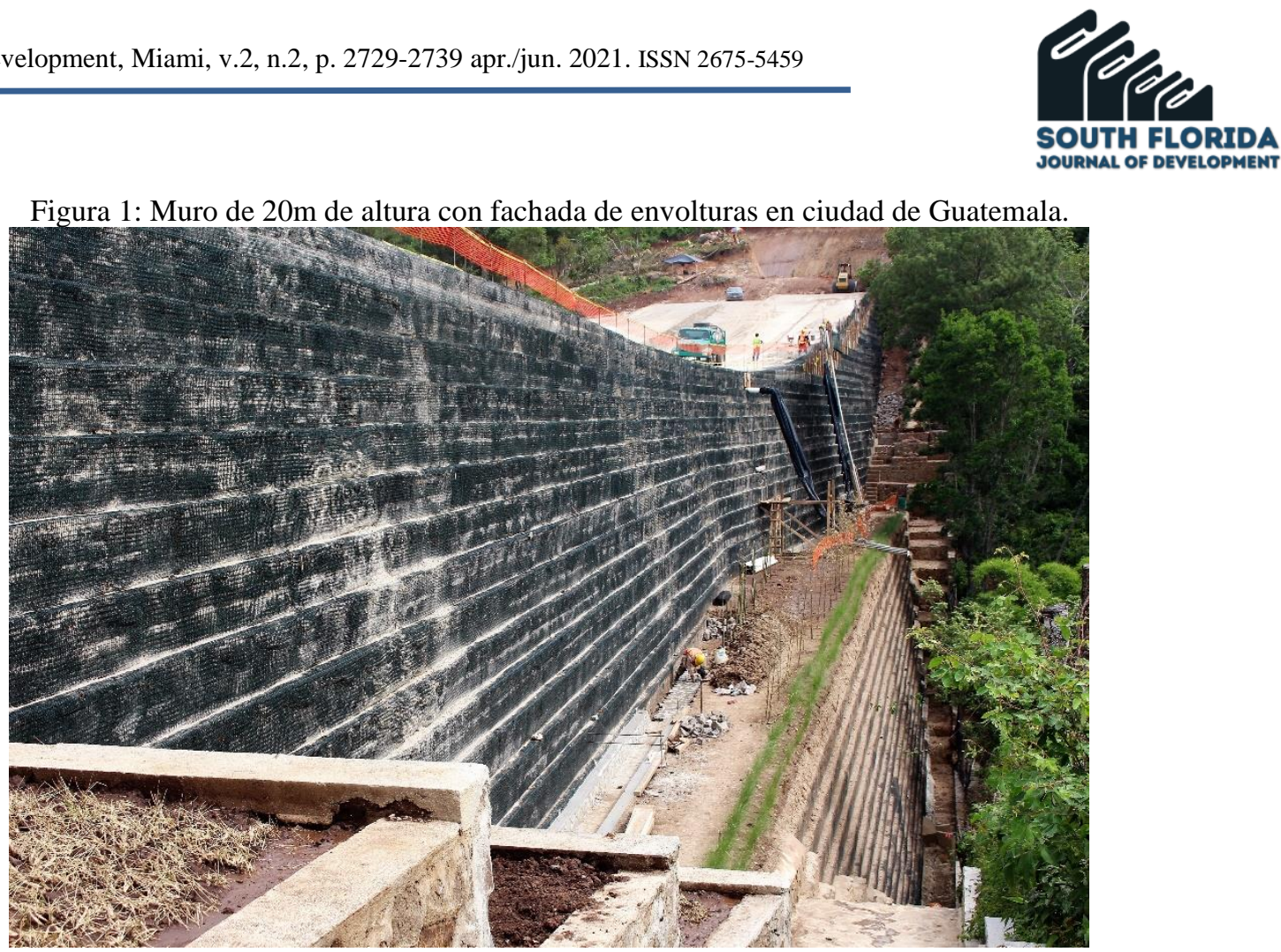

La geometría del muro en la zona de mayor altura desarrolló una sección inferior de 11 metros de altura y una superior de 9 metros. El muro se cimentó en el fondo de una quebrada de invierno por lo que fue necesario construir un sub-drenaje de grandes dimensiones, el cual ha funcionado hasta la fecha de manera adecuada.

Con una geometría de altura variable y tratando de minimizar el impacto del muro al entorno ecológico de la zona, se propuso el uso de una fachada de envolturas de geomalla biaxial de polipropileno y mantos de control de erosión con protección adicional contra rayos ultravioleta.

Debido a la geometría y distancia entre las caras de las dos secciones, el muro se diseñó como un muro compuesto. La longitud de las geomallas en la sección inferior llegó a los 11.80 metros y en la sección superior a los 6.40 metros. La separación vertical de las geomallas se encuentra entre $10 \mathrm{c} 20 \mathrm{~cm}$ y $60 \mathrm{~cm}$. Las envolturas tienen una altura de $60 \mathrm{~cm}$ y una conexión por fricción 1.20 metros dentro del relleno como lo indica la normativa FHWA NHI-00-043, para este tipo de estructura (FHWA,2001).

Se desarrolló un estudio de suelos que llenara lo establecido por la normativa nacional de AGIES 2.1-2010 (AGIES, 2010), así como un diseño que llevó un análisis de estabilidad interna, externa, compuesta y global según lo establecido por AASHTO en su "Standard Specifications for Highways 17 th. Edition, 2002" (AASHTO, 2002) y cumpliendo con los empujes y carga sísmica que establece la norma AGIES 5.3-2010 (AGIES, 2010).

Para el relleno se utilizó material obtenido de los cortes del propio proyecto lo que le ayudó a ser económicamente viable. El relleno compuesto de arenas limosas de buena capacidad mecánica con ángulos de fricción interna de $36^{\circ}$, reducido a $34^{\circ}$ para diseño, cohesión de $25 \mathrm{kPa}$, la cual fue reducida a 
$5 \mathrm{kPa}$ y una densidad de $17 \mathrm{kN} / \mathrm{m} 3$. Como suelo de cimentación se utilizaron estratos de limos arcillosos con ángulos de fricción alrededor de los $32^{\circ}$, cohesión de $30 \mathrm{kPa}$ y densidad de $17 \mathrm{kN} / \mathrm{m} 3$.

Se modelaron dos sobrecargas en la corona del muro, en los primeros 10 metros se colocó una carga distribuida de $10 \mathrm{kPa}$ y a 20 metros de distancia de la corona una de $20 \mathrm{kPa}$, la primera modela la carga de una calle de acceso y la segunda una futura construcción.

Como una protección al pie del muro y como un sistema de drenaje se construyó una cuneta de zampeado de piedra y un cabezal de concreto para la salida de la tubería del sub-drenaje. La obra se inició a principios del 2016 y se llevó 7 meses finalizarla.

Todos los materiales geosintéticos fueron fabricados en Estados Unidos de América, se necesitaron más de 30,607 $\mathrm{m} 2$ de geomalla uniaxial de polietileno de alta densidad, 14,250 m2 de geomalla biaxial de polipropileno y $8,580 \mathrm{~m} 2$ de manto de control de erosión permanente de polipropileno.

\subsection{MUROS DE RETENCIÓN DE SUELO REFORZADO DE 60 M (ESCALONADOS) EN}

\section{FUJAIRAH, EAU}

Los RSRW se introdujeron por primera vez en los principales proyectos de ingeniería civil en los Emiratos Árabes Unidos (EAU) alrededor del año 2000. El proyecto que impulsó esta tendencia fue el Dibba Idhn Tawaian Road, Dubai. Con una superficie total de más de 40,000 m2, introdujo los sistemas RSRW en los Emiratos Árabes Unidos demostrando que son muy adecuados para la construcción a través de terrenos montañosos difíciles y en condiciones climáticas extremas. El más impresionante es el Proyecto de la Autopista de Dubai Fujairah para el Ministerio de Obras Públicas, con el área total que supera los 100,000 m2., es uno de los muros más altos jamás construido con geomallas de HDPE.

Con el fin de facilitar el desarrollo de la región, el Ministerio de Obras Públicas de los Emiratos Árabes Unidos decidió construir una autopista de 4 carriles (10 carriles en total, incluidos 2 de hombros), que unirá Fujairah con Dubai. Cuando se complete, esta autopista tendrá una longitud de 80 km y se espera que se disminuya a la mitad el tiempo de viaje entre Dubai y Fujairah.

El área total construida de muro de suelo reforzado supera los $100.000 \mathrm{~m} 2$ y comprende 29 muros individuales, que comprenden muros sencillos, así como estructuras complejas escalonadas configuradas de la siguiente manera:

- Muros sencillos con un ángulo de cara de $86^{\circ}$ y hasta $22 \mathrm{~m}$ de altura

- Muros de 2 secciones que comprenden dos muros con una berma intermedia que varía de 5 a $15 \mathrm{~m}$, formando la elevación general de la estructura con una altura total de hasta $40 \mathrm{~m}$.

- Muros de 3 secciones que comprenden tres muros con tres bermas horizontales que van desde 5 a $20 \mathrm{~m}$, formando la elevación general de la estructura con una altura total de hasta $60 \mathrm{~m}$. 
Figura 2: Muro de 60m de altura total, Fujairah, Emiratos Árabes Unidos.

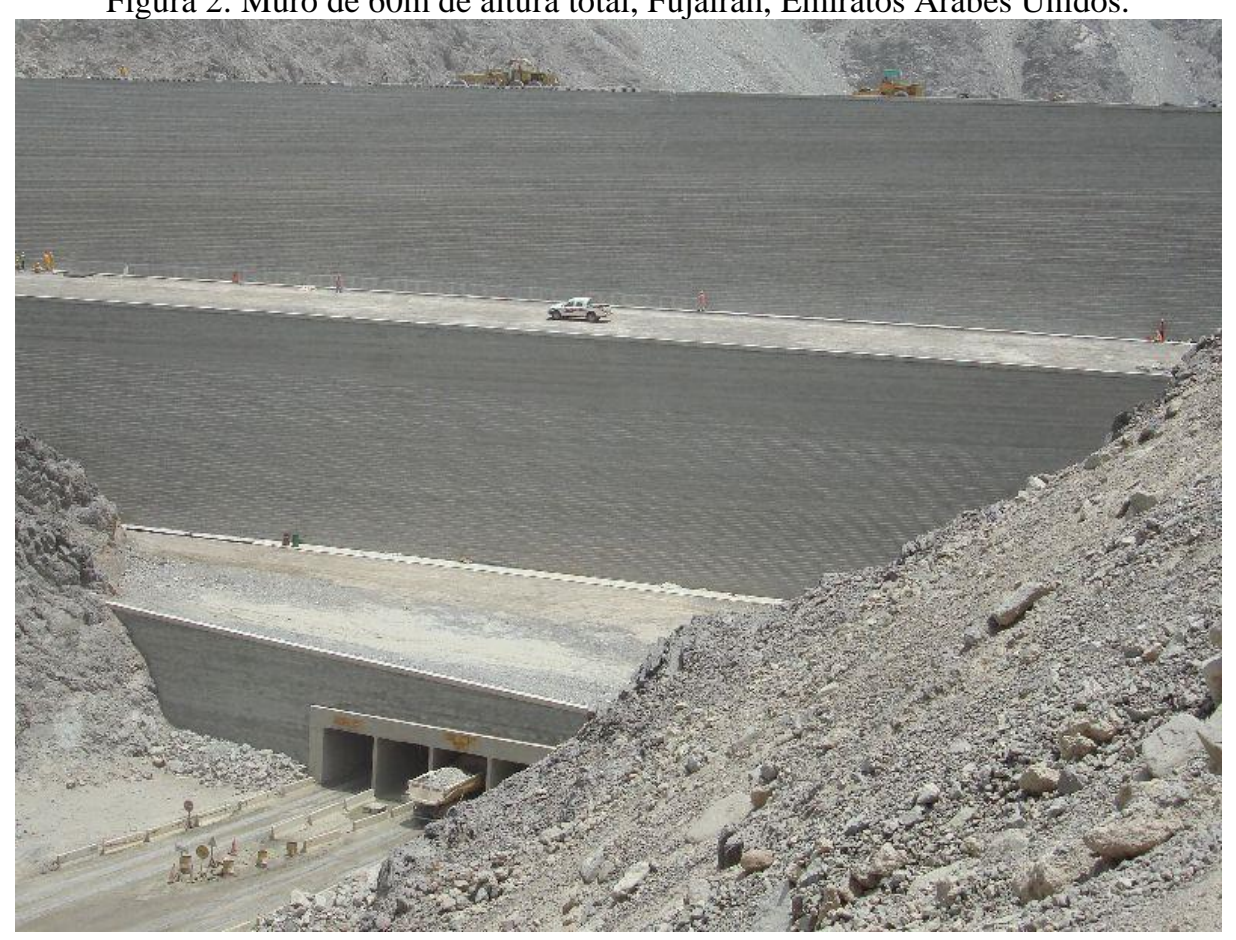

Todos los muros se construyeron con unidades de bloques de concreto con una resistencia mínima a la compresión a los 28 días es de $30 \mathrm{MPa}$. Tanto las geomallas de HDPE de orientación uniaxial de Tensar como el conector de revestimiento mecánico se produjeron en el Reino Unido y se transportaron a los EAU.

El proyecto fue una importante operación de corte y relleno, lo que hizo que el uso del muro de retención de suelo reforzado fuera tan atractivo. Todo el material de relleno reforzado se obtuvo localmente en Gabbro, producido a partir de voladura, luego se trituró y clasificó al tamaño máximo de partícula de $37.5 \mathrm{~mm}$. Se sometieron a múltiples pruebas muestras de este material en cajas de corte de acuerdo con la guía de los estándares británicos sobre identificación y descripción de suelos (British Standards Institution, 1995). Se encontró que el ángulo de fricción interna era superior a $\phi=45^{\circ}$. Se adoptó un valor máximo conservador (pico) de $\phi \mathrm{pk}^{\prime}=42^{\circ}$ para el diseño. Se usó material de relleno similar, pero de calidad ligeramente menor como relleno detrás de la zona de suelo reforzada con un valor de diseño de ángulo de fricción interna (pico) de $\phi \mathrm{pk}^{\prime}=40^{\circ}$.

Aunque la mayoría de los muros se cimentaron en roca, había áreas donde los muros se cimentaban en los wadis (nombre local a las quebradas de invierno) existentes, donde los suelos comprendían depósitos aluviales sueltos en la superficie. Bajo una estricta supervisión y como parte de la responsabilidad del contratista, cualquier depósito de este tipo fue removido y reemplazado por relleno de roca diseñado para proporcionar una base competente para los muros. 
Los diseños de los muros sencillos se llevaron a cabo utilizando el principio de diseño de cuña de 2 partes. Los muros compuestos de 2 niveles y 3 niveles se analizaron como estructuras compuestas y teniendo en cuenta el efecto de los niveles superiores sobre los niveles inferiores y viceversa, cuando corresponda. Los muros se modelaron por primera vez individualmente con el nivel superior modelado como sobrecarga y finalmente, toda la estructura de múltiples niveles se modeló en un software de estabilidad y se analizó con el método simplificado de Bishop para la estabilidad interna, compuesta y global.

Se modeló una sobrecarga temporal de $20 \mathrm{kPa}$ en la corona de todos los muros para modelar el tránsito de la autopista en el futuro. El agua subterránea se modeló para atender las crecidas repentinas durante la temporada de fuertes lluvias que azotan en los Emiratos Árabes Unidos.

Las geomallas se colocaron con sus costillas y aberturas perpendiculares a la fachada y se aseguraron al bloque modular con un conector mecánico de polímero. Los bloques modulares de concreto de $200 \mathrm{~mm}$ de ancho, $200 \mathrm{~mm}$ de profundidad y $400 \mathrm{~mm}$ de largo se instalaron sin utilizar mortero, lo que eliminó la necesidad de utilizar productos a base de agua en el proceso. El relleno se colocó y compactó en capas $200 \mathrm{~mm}$ de espesor. La geomalla se colocó entre las capas de relleno compactadas a una separación vertical que varía de 200 mm a 600 mm.

Después de la compactación, cada capa de relleno se ensayó en el sitio para garantizar que se lograra una compactación de por lo menos el 95\% de su densidad seca.

Se construyeron de 100 a $150 \mathrm{~m} 2$ de muro diariamente. Al terminar los muros se colocaron protecciones con cajas de gavión en la parte inferior para evitar los daños asociados a la socavación que pudieran causar las crecidas.

\section{CONCLUSIONES}

En los últimos 40 años, las estructuras reforzadas con geomalla polimérica se han establecido como alternativas confiables a las estructuras convencionales de concreto reforzado. Se han publicado varios métodos de diseño validados para respaldar los principios de diseño asociados con el uso de refuerzo de geomallas poliméricas, y se utilizan ampliamente en todo el mundo. En muchas situaciones, el uso de geomallas poliméricas ha abierto posibilidades para la construcción de estructuras extraordinarias que de otro modo no serían factibles o serían extremadamente costosas. Como ejemplos mencionamos los muros de suelo reforzado con polímeros de $60 \mathrm{~m}$ de alto que se utilizaron como alternativas a los viaductos de concreto convencionales en Fujairah, EAU y muros de $20 \mathrm{~m}$ de alto de pantalla flexible de envolturas en Guatemala. 
Tanto los muros con fachada de bloque modular como los sistemas de muros de envolturas presentados en este documento han demostrado ser muy exitosos, ya que permiten una construcción muy rápida y ofrecen soluciones que resultan en estructuras atractivas, estables, rentables y de bajo mantenimiento durante sus 120 años de vida de diseño.

\section{RECONOCIMIENTO}

Los autores agradecen sinceramente a los propietarios y diseñadores de ambos proyectos por permitir la publicación y a los colegas de Tensar e Ingnova por toda su valiosa ayuda y apoyo en la redacción y edición de este documento. 


\section{BIBLIOGRAFÍA}

AASHTO Standard Specifications for Highways 17 th. Edition, 2002.

AGIES, 2010. Normas Estructurales de Diseño y Construcción Recomendadas para la República de Guatemala, 2010. NR-1 Bases Generales de Diseño, Construcción.NR-2.1 Estudios geotécnicos y NR5.3 Obras de retención.

BBA Certificate No. 99/R109, 1999: Tensar RE and RE500 Geogrids for Reinforced Soil Wall and Bridge Abutments Systems, British Board of Agrément, Watford, UK

British Standards Institution, 1995, revised 2010, Code of practice for strengthen/reinforced soils and other fills, BS 8006, HMSO, London

C. Doulala-Rigby, J. Dixon, "Use of Site Won Chalk for the construction of steep Geogrid Reinforced Soil Embankments in the South of England, UK" - presented and published in the 15th European Conference on Soil Mechanics and Geotechnical Engineering, Athens, Greece, September 2011

C. Doulala-Rigby, M. Black, 2015, "The design and construction of a bridge approach embankment utilising mechanically stabilised earth walls with geogrid reinforced pulverised fuel ash fill" - presented and published in $3^{\text {rd }}$ Pan-American Conference on Geosynthetics, Miami, USA, 2015

C. Doulala-Rigby, S. Karri, R Branford, 2016, "The use of polymeric geogrids with light weight aggregate fill" - presented and published in the $19^{\text {th }}$ International Conference on Soil Mechanics and Geotechnical Engineering, Seoul, South Korea, 2017

C. Doulala-Rigby, A. Stone, "Landfill slip failure repair with geogrids using waste fill material at Danylan, Wales, UK" - presented and published in the 14th Asian Regional Conference on Soil Mechanics and Geotechnical Engineering, Hong Kong, China May 2011; also presented and published in the 2nd World Landslide Forum, Rome, Italy, October 2011

C.J.F.P. Jones, C. Doulala-Rigby, 2014, “The first polymeric geogrid reinforced soils structure" published in the $10^{\text {th }}$ International Conference on Geosynthetics, Berlin, Germany, 2014

FHWA,1993. Guidelines for Design, Specification, and Contracting of Geosynthetic Mechanically Stabilized Earth Slopes on Firm Foundations.

FHWA,2001. Publication NHI-00-043, Mechanically Stabilized Earth Walls and Reinforced Soil Slopes Design an 